\title{
Simplified cell culture method for the diagnosis of atypical primary ciliary dyskinesia
}

\author{
M Pifferi, ${ }^{1}$ F Montemurro, ${ }^{2}$ A M Cangiotti, ${ }^{3}$ V Ragazzo, ${ }^{1}$ M Di Cicco, ${ }^{1}$ B Vinci, ${ }^{2}$ G Vozzi, ${ }^{2}$ \\ P Macchia, ${ }^{1}$ A L Boner ${ }^{4}$
}

- Additional methods and video clips are published online only at http://thorax.bmi.com/content/ vol64/issue12

${ }^{1}$ Department of Pediatrics, University of Pisa, Italy;

${ }^{2}$ Interdepartmental Research Center "E. Piaggio", Faculty of Engineering, University of Pisa, Italy: ${ }^{3}$ Institute of Normal Human Morphology, Electron Microscopy Unit, Umberto ${ }^{\circ}$ Hospital, University of Ancona, Italy; ${ }^{4}$ Department of Pediatrics, University of Verona, Italy

Correspondence to: Dr M Pifferi, Department of Pediatrics, University of Pisa, Via Roma 67, 56126 Pisa, Italy; m.pifferi@med.unipi.it

Received 11 November 2008 Accepted 2 September 2009 Published Online First 21 September 2009

\section{ABSTRACT}

Background: The diagnosis of primary ciliary dyskinesia (PCD) can be challenging, and it may be particularly difficult to distinguish primary ciliary disease from the secondary changes after infections.

Objectives: The purpose of the study was to evaluate if nasal epithelial cells, obtained with nasal brushing instead of a biopsy, could be used in a culture system for the diagnosis of PCD in difficult cases.

Methods and main results: Ciliary motion analysis (CMA) and transmission electron microscopy (TEM) were performed on 59 subjects with persistent or recurrent pneumonia. These investigations allowed the diagnosis of PCD in $13(22 \%)$ patients while the defect of the cilia was considered secondary to infections in $37(63 \%)$ subjects. In the remaining nine (15\%) patients the diagnostic evaluation with CMA and TEM remained inconclusive. Ciliogenesis in culture allowed the diagnosis of PCD in four of these patients, it was indicative of a secondary defect in two subjects, and it was not helpful in the remaining three patients.

Conclusions: Culture of cells obtained with brushing of the nasal turbinate is not a perfect test, nevertheless it may offer diagnostic help in doubtful cases of PCD.

Primary ciliary dyskinesia (PCD) is a phenotypically and genetically heterogeneous disorder with an autosomal-recessive inheritance pattern. It is a rare and difficult to diagnose disease, ${ }^{12}$ with morbidity related to recurrent infections of the respiratory tract. These often result in development of bronchiectasis mainly in relation to the frequent delay in disease recognition. ${ }^{3}{ }^{4}$ Diagnosis currently relies on clinical features together with distinctive transmission electron microscopy (TEM) images, and typical ciliary beat frequency and pattern phenotypes at digital high-speed video imaging recording. ${ }^{5}$ Even with these demanding techniques the diagnosis of PCD may be not easy and may require repeated testing in doubtful cases.

Difficulties and uncertainties are related first to the fact that anatomical findings may be secondary to infection/inflammation; secondly, in a few patients with immotile cilia, a normal ciliary ultrastructure might be found, suggesting that abnormal ciliary motion could also be related to cellular defects not detectable by ultrastructural study. ${ }^{6-9}$ Finally, another subset of patients with symptoms of PCD demonstrate not only normal ciliary structure, but also an apparently normal motility but with a random orientation of the cilia which results in ineffective and/or impaired mucociliary clearance. ${ }^{10}$
In these conditions genetic analysis would represent the ideal solution, but PCD is genetically heterogeneous, being caused by mutations in a number of different genes which have not yet all been discovered. ${ }^{11}$ Nasal nitric oxide (nNO) is helpful in the evaluation, usually being low even in atypical PCD. ${ }^{12}$ However, $\mathrm{nNO}$ may be normal in a minority of patients with $\mathrm{PCD}^{2}$ and it may be low in subjects with other conditions such as, for example, sinusitis, upper airway infections ${ }^{13}$ and cystic fibrosis. ${ }^{14}$

In these difficult situations the diagnosis can be reached with the culture of ciliated cells in sequential monolayer-suspension since all secondary damage is virtually absent after regrowth of the ciliated epithelium in culture while primary defects remain unaffected. ${ }^{15}{ }^{16}$ However, a number of limitations and disadvantages of cell culture systems have been reported. In particular, a biopsy of nasal turbinate is needed to start the culture, since brushings and scrapings were previously considered not to give sufficient material, with up to $50 \%$ of inconclusive investigations ${ }^{17}$; however, this procedure induces epistaxis and pain. Furthermore, the primary culture system using biopsy material is complicated and technically demanding.

For these reasons the aim of our study was to assess if nasal brushing could be used when a simplified cell culture system ${ }^{18}$ is applied in the diagnostic investigation of difficult to diagnose patients with PCD.

\section{MATERIALS AND METHODS \\ Subjects}

Fifty-nine consecutive subjects with a clinical history of recurrent pneumonia, defined as at least two pneumonia episodes in 1 year or $>3$ at any time, ${ }^{19}$ were evaluated in the Department of Pediatrics, University of Pisa, a National Referral centre, to rule out PCD. Pneumonia was defined as the presence of fever and acute respiratory symptoms with crackles on auscultation. Evidence of concomitant parenchymal infiltrates on chest radiography was required on at least two occasions. The evaluation was performed when subjects were free from acute respiratory infection for at least 3-4 weeks.

Patients were required to stop any pharmacological treatment $48 \mathrm{~h}$ prior to the evaluation. Ciliary motion analysis and ultrastructural assessment were performed on nasal brushing samples. Concomitantly, nasal epithelial cells obtained in the same nasal brushing procedure were cultured for ciliary functional analysis after ciliogenesis. A 
second ciliary motion analysis and ultrastructural assessment was only performed in subjects with inconclusive results after the first evaluation.

Informed consent for nasal brushing was obtained from the children's parents, and the Hospital Ethical Committee approved the study protocol.

\section{Nasal sampling}

Samples, obtained from the inferior turbinate using a cytology brush (Microvasive, Milford, Massachusetts, USA), were suspended in $2 \mathrm{ml}$ of Medium 199 fluid cell culture, in $2 \mathrm{ml}$ of normal saline or in $5 \mathrm{ml}$ of a phosphate-buffered saline (PBS)/ $2 \%$ antibiotic solution, for immediate light microscopic evaluation, for TEM evaluation and for cell cultures, respectively. ${ }^{20}$ Ciliary motion analysis, ultrastructural assessment and cultures were performed by different operators, each one blind to the results obtained by the others.

\section{Ciliary motion analysis and ultrastructural studies}

Ciliary morphology, motion pattern, beat frequency and ultrastructural studies were evaluated according to standardised methodology. ${ }^{21-25}$ Subjects were classified as having PCD or secondary ciliary dyskinesia (SCD) on the basis of structural and functional ciliary abnormalities. ${ }^{12} 2223$ In the case of inconclusive functional or TEM evaluations, patients had to be considered borderline (fig 1) and a second evaluation was performed 4-6 months later, after appropriate antibiotic and anti-inflammatory treatment ( $\beta$-lactam compounds in combination with $\beta$-lactamase inhibitors and azithromycin) to try to distinguish between primary and secondary problems. ${ }^{1}{ }^{23}$

\section{Nasal nitric oxide}

$\mathrm{nNO}$ was evaluated according to suggested methodology. Nasal air was sampled continuously with a constant transnasal flow of $1.8 \mathrm{ml} / \mathrm{s}$ for $\geqslant 30 \mathrm{~s}$ (CLD 88 Exhalyzer; EcoPhysics, Duernten, Switzerland). ${ }^{26}$

\section{Suspension cell cultures}

The cell suspension used for cell cultures was centrifuged at $1500 \mathrm{rpm}$ for $7 \mathrm{~min}$ to remove PBS. The pellet was resuspended in $4 \mathrm{ml}$ of medium (see culture medium section for details) and cells were seeded in well culture plates previously coated with $1 \%$ gel. After $24 \mathrm{~h}$ samples were moved to another well to eliminate adherent cells and erythrocytes. Cultures were incubated at $37^{\circ} \mathrm{C}, 5 \% \mathrm{CO}_{2}$, adding $1 \mathrm{ml}$ of fresh medium every 3 days and changing the medium once a week. Ciliogenesis of human respiratory epithelium takes place when aggregated cells are floating through the medium in the form of spheroids. ${ }^{18} 27$ After ciliogenesis in culture continuous movements (migrations and/or rotations) of the spheroids are considered to be the expression of coordinated ciliary activity. ${ }^{17}{ }^{18}$ As previously reported, ${ }^{18}$ by this technique spheroids can be identified after $24-48$ h of culture but, in our protocol, the evaluation of ciliary activity was performed on the 21st day of culture when new cilia formation had definitely occurred. ${ }^{27}$

\section{Culture medium}

For cell cultures we used Dulbecco's modified Eagle's medium (DMEM)/nutrient mixture F-12 Ham 1:1 (Sigma, Milan, Italy) supplemented with 10\% (v/v) fetal bovine serum (FBS), $2 \%$ (v/ v) L-glutamine, $2 \%(\mathrm{v} / \mathrm{v})$ antibiotic/antimycotic solution, $5 \mu \mathrm{g} /$ $\mathrm{ml}$ insulin, $100 \mu \mathrm{g} / \mathrm{ml}$ hydrocortisone, $30 \mathrm{ng} / \mathrm{ml}$ epidermal growth factor (EGF), $2 \mu \mathrm{g} / \mathrm{ml}$ endothelial cell growth supplement from bovine pituitary (ECGS), 200 pM triiodo-L-thyronine and $10 \mu \mathrm{g} / \mathrm{ml}$ transferrin.

\section{Ciliary activity evaluation after ciliogenesis in culture}

Spheroids were identified using inverse microscopy (Inverted Microscope TE2000-S, Nikon, Tokyo, Japan) at $\times 60$ and images were analysed every 3 days (see video clips in the online data supplement). Absence of coordinated ciliary activity or ciliary immotility on the 21st day of culture was considered diagnostic for PCD..$^{18}{ }^{28}$ Additional details of the method are provided in the online data supplement.
Figure 1. Simplified diagnostic algorithm for atypical primary ciliary dyskinesia $\left({ }^{*}\right.$ nasal nitric oxide evaluations might be of help in the diagnostic process).

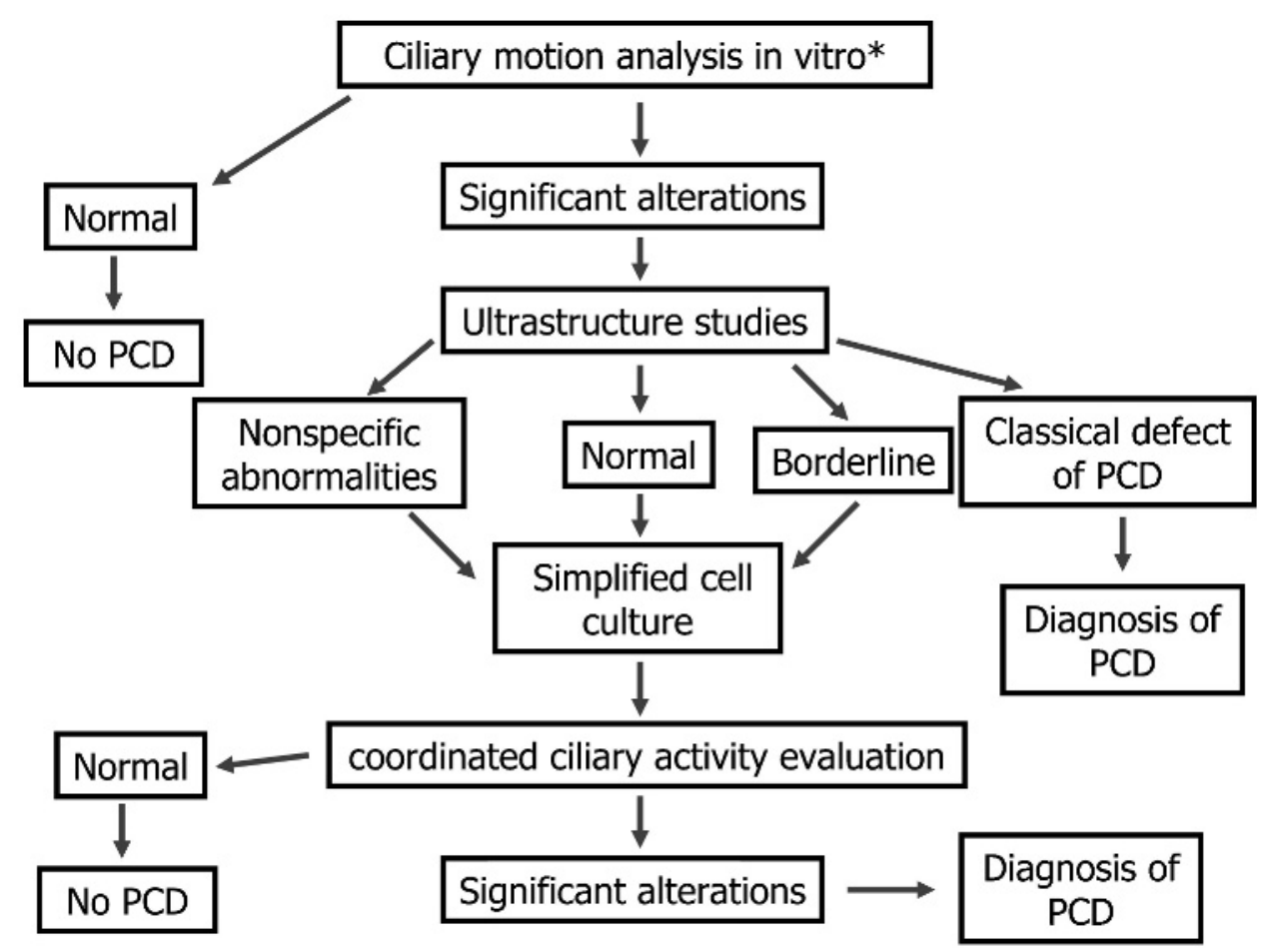




\section{Statistical analyses}

Baseline patient characteristics are reported as group mean (SD). Medians for $\mathrm{nNO}$ were considered. A $\mathrm{p}$ value $<0.05$ was considered statistically significant. All statistical calculations were performed using SPSS version 14.0 software for Windows (SPSS, Chicago, Illinois, USA) for personal computers. ${ }^{29}$

In the absence of a true gold standard test, the diagnosis obtained, at the end of the diagnostic pathway as described above (fig. 1), by ciliary motion analysis, TEM evaluation and based on ciliary activity evaluation after ciliogenesis in culture, was accepted. ${ }^{17}$

\section{RESULTS}

Of 59 subjects (28 males) studied, 42 were children (age range: 3 months-17.5 years, mean (SD) 8.8 (4.7) years) and 17 were adults (age range: 20.8-57.4 years, mean (SD) 34.9 (12.1) years).

Immunological abnormalities were excluded in all patients. No subject had cystic fibrosis or cystic fibrosis gene mutations (33 mutations have been sought), and careful examination allowed us to exclude swallowing problems and clinical signs of supraoesophageal complications of gastro-oesophageal reflux.

In 20 children brushing was repeated a second time, for ciliary motion analysis and ultrastructural assessment, because of equivocal results at the first examination.

Ciliary motion analysis (abnormal motion patterns, including immotile cilia and/or very low ciliary beat frequency) and TEM evaluation of cilia (alterations of the central pair and deficiencies of the dynein arms, associated with a small proportion of swollen cilia and compound cilia) confirmed the diagnosis of PCD in 13 (7 children, 6 adults) subjects (22\%); in two patients the diagnosis was reached with the second sampling. In those 13 patients the median $\mathrm{nNO}$ was 64.9 parts per billion (ppb) and the range was 9.1-1000 ppb; in 2 patients the value of $\mathrm{nNO}$ was $>200 \mathrm{ppb}$. Epithelial cell culture confirmed the diagnosis of PCD in 10 patients (see online video 1); in the remaining 3 subjects this was not possible because the cultures became infected. According to the clinical phenotype, 8 of these 13 patients had Kartagener syndrome.

In another $37(62.7 \%)$ patients (27 children, 10 adults), ciliary motion analysis demonstrated abnormal patterns in a small proportion of cilia, prevalence of thick cilia and low ciliary beat frequency, compatible with SCD (in 9 of them the diagnosis was reached after the second sampling). In these 37 subjects, TEM evaluation clearly showed non-specific abnormalities compatible with chronic inflammation (prevalence of swollen cilia and compound cilia). In this group the median nNO was $550.2 \mathrm{ppb}$ and the range was $32.7-1000 \mathrm{ppb}$ (in one subject $\mathrm{nNO}$ was $<200 \mathrm{ppb}$ ), and epithelial cell culture confirmed the secondary nature of the lesions in 30 subjects and failed in 7 subjects ( 4 infected cell culture, 3 borderline-that is, ambiguous migration and/or rotations: see video 2 in the online supplement). In the 3 patients with a borderline culture, $\mathrm{nNO}$ values were $579.9,576.7$ and $77.6 \mathrm{ppb}$, respectively.

In the remaining $9(15.2 \%)$ subjects ( 1 with dextrocardia, 3 with situs viscerum inversus) functional or TEM evaluations were inconclusive (borderline), even when the investigation was repeated after intensive and prolonged treatment with antibiotics. In those patients the median nNO was $466.6 \mathrm{ppb}$, range $19.8-814.8 \mathrm{ppb}$ (in 5 patients $>200 \mathrm{ppb}$ ). However, in 4 out of these 9 patients classified as borderline (44.4\%) functional ciliary evaluation after ciliogenesis in culture allowed a firm diagnosis of PCD in relation to complete absence of either migration or rotation of spheroids (see video 3 in the online supplement). In these 4 patients the individual results for $\mathrm{nNO}$ were 19.8, 26.9, 33.7 and $466.6 \mathrm{ppb}$. Furthermore, the culture was indicative of SCD in 2 cases with $\mathrm{nNO}$ values of 814.8 and $794.7 \mathrm{ppb}$ and did not help diagnostically in 3 subjects (1 infected cell culture, 2 borderline). In these 3 patients who remained undiagnosed, individual $\mathrm{nNO}$ results were 725.5, 64.3 and $576.7 \mathrm{ppb}$.

Thus the nasal epithelial cell culture procedure was successful in confirming or excluding the diagnosis of PCD in 46 out of 59 cases (78\%); 8 cultures became infected and were unusable. Unfortunately, the cultures were borderline in 5 (9\%) cases, since a coordinated activity or, on the contrary, complete immotility was not observed. However, in 3 out of these 5 cases ciliary motion analysis and TEM evaluation already had provided a diagnosis of SCD. Therefore, combining the results of all the investigations, the diagnosis of PCD was established in 17 subjects, the diagnosis of SCD was obtained in 39 subjects, and only 3 patients were classified as borderline (table 1 ).

\section{DISCUSSION}

We have shown that nasal epithelium cell culture is also possible from cells obtained with nasal brushing. A successful culture in nearly $80 \%$ of the samples is clearly superior to the $50 \%$ previously reported ${ }^{17}$ and is similar to that reported from cultures of samples obtained from a nasal turbinate biopsy. ${ }^{28}$ Culture from brushings ${ }^{18}$ instead of a biopsy ${ }^{15-17} 283^{30}$ has several advantages besides that of easier sampling. Epithelial cells do not need to be dissociated from the tissue with proteases, require fewer suspension-centrifugation-resuspension passages which reduces the risk of cellular damage, and do not need to be placed on thick collagen. Furthermore, the culture medium does not have to be replaced each day but only once a week, and the formation of stable aggregates (epithelial spheroids) of ciliaproducing cells occurs earlier. All these aspects reduce the technical complexity of the procedure which has the same specificity $(100 \%)$ claimed in a review on the topic of cultures of cells obtained with a turbinate biopsy. ${ }^{31}$ Furthermore, starting the culture directly from the brushed material may reduce the need for a second sampling after a period of antibiotic and antiinflammatory treatment as suggested in fig 1. A slightly similar approach for the culture of airway epithelial cells was previously followed by Toskala et al in patients with chronic sinusitis. ${ }^{32}$ This group performed a simple brush after pretreatment with cocaine and adrenaline, and the samples had to be digested with pronase in order to remove fibroblasts. Furthermore, large mucosal specimens were not taken from controls for ethical reasons, suggesting theirs is a more invasive technique. Our method, not previously attempted, has the advantage of being simple and non-invasive, and can give an answer after 3 weeks.

The culture of ciliated cells is only necessary in the case of doubt and is not obligatory for the diagnosis of patients with clear-cut PCD. However, on occasions the diagnosis of PCD may be difficult due to damage of the respiratory epithelium secondary to infection or inflammation, or there may be near normal TEM despite alteration in ciliary beat patterns strongly suggestive of a primary disease. If secondary damage is suspected a second sampling is usually recommended after a period of antibiotic and anti-inflammatory therapy. Nevertheless, in our study population, this was still not diagnostic in $\sim 15 \%$ ( 9 out of 59 ) of the patients. The unsuccessful outcome can be explained either by non-adherence to the suggested regimen, to colonisation with resistant microorganisms or to the long-lasting nature of the infection/ inflammation processes. 
Table 1 Diagnostic results in patients with ciliary dyskinesia

\begin{tabular}{llccl}
\hline \multirow{2}{*}{ Diagnosis by CMA and TEM } & \multicolumn{4}{l}{ Diagnosis by ciliogenesis in culture } \\
\cline { 2 - 5 } & No. of samples $\dagger$ & PCD & SCD & Borderline \\
\hline PCD (13)\$ & 10 & $10(100 \%)$ & $0(0 \%)$ & $0(0 \%)$ \\
SCD (37)§ & 33 & $0(0 \%)$ & $30(90.9 \%)$ & $3(9.1 \%)$ \\
Borderline (9) & 8 & $4(50 \%)$ & $2(25 \%)$ & $2(25 \%)$ \\
\hline
\end{tabular}

* The number of subjects is given in parentheses.

† In three subjects with PCD, in four with SCD, and in one defined as borderline the cell cultures were found to be infected and consequently not useful.

In two subjects the diagnosis was reached with a second sampling.

§In nine subjects the diagnosis was reached with a second sampling,

In nine subjects the diagnosis was also inconclusive with a second sampling.

CMA, ciliary motion analysis; PCD, primary ciliary dyskinesia; SCD, secondary ciliary dyskinesia; TEM, transmission electron microscopy.

If the diagnosis is difficult, or if the patient is suspected of having an unusual PCD phenotype, which has been reported to be the case in as many as $25 \%$ of patients, ${ }^{28}$ culture of ciliated cells provides additional information. Effectively, secondary damage is virtually absent after ciliogenesis in a suspension culture so that if a frank abnormal pattern of ciliary movement persists after culture this has to be considered to be primary. ${ }^{16}$

In our study, cultures obtained from three patients with presumed SCD remained borderline-that is, a coordinated activity or, on the contrary, complete immotility was not observed. In these subjects further testing, including possibly genetic testing, is required. Furthermore, one of these patients had $\mathrm{nNO}<200 \mathrm{ppb}$, which may indicate a primary defect.

In general, in our study population, adding $\mathrm{nNO}$ in the diagnostic algorithm of ciliary dysfunction diseases was misleading (>200 ppb) in 3 out of $17(17.6 \%)$ patients with PCD, and was abnormally low $(<200 \mathrm{ppb})$ in one patient out of $39(2.6 \%)$ with the final diagnosis of SCD and in 1 out of 3 patients who remained without a definitive diagnosis also after ciliogenesis in culture. For these reasons we agree with Bush et al that this evaluation is helpful for the selection of patients for brush biopsy and that if a repeated brushing fails to yield an answer, nNO may be a very helpful diagnostic pointer ${ }^{33}$; however, we would like to stress that, until the genetics of the disease are completely worked out, some patients will remain a diagnostic puzzle. Indeed, despite investigations three patients remained undiagnosed, and one of these had $\mathrm{nNO}$ $<200 \mathrm{ppb}$ suggesting a possible diagnosis of PCD. Again, whether a culture of respiratory epithelial cells obtained with a turbinate biopsy would solve the diagnostic dilemma in these three patients is unknown. In the meantime we recommend that the follow-up and treatment for those subjects should be the same as that of subjects with PCD, with regular physiotherapy and prompt and aggressive use of antibiotics in the case of exacerbations in order to prevent further airway damage.

Our study has at least two limitations. First, electron microscopy was not performed in cultured cells. This would strengthen our findings, but it was not possible because of the small amount of tissue present in the cultures. Secondly, the sensitivity, specificity, and the positive and negative predictive values of ciliogenesis in culture could not be calculated using the gold standard of cases with an independent genetic diagnosis, but this is actually not possible in clinical practice.

Culture of respiratory epithelial cells obtained with brushing is not a perfect test, mainly because of the possibility of infection leading to loss of the culture, but it may offer diagnostic help in doubtful cases of PCD. It has the further advantage of ease of performance and less trauma than cup forceps biopsy of the turbinates.
In conclusion, the diagnosis of PCD should be performed on the basis of ciliary function analysis and TEM, which are the gold standards in diagnosis by current methods. In light of there being insufficient material for both ciliary function analysis and TEM following culture this approach cannot be used in all patients. However, if ciliary function analysis, a test which is immediately available, is suggestive for the disease and if the patients lives far away from the centre, a cell culture may be considered concomitantly in a single diagnostic session.

Acknowledgements: We are grateful to Professor Andrew Bush for his generous help in the discussion and for the final editing of the manuscript.

Funding: Fondazione Cassa di Risparmio di Pisa, Italy.

Competing interests: None.

Ethics approval: Ethics approval for this study was obtained from the Hospital Ethics Committee of Pisa.

Provenance and peer review: Not commissioned; externally peer reviewed.

\section{REFERENCES}

1. Bush A, Cole P, Hariri M, et al. Primary ciliary dyskinesia: diagnosis and standard of care. Eur Respir J 1998;12:982-8.

2. Bush A, Chodhari R, Collins N, et al. Primary ciliary dyskinesia: current state of the art. Arch Dis Child 2007;92:1136-40.

3. Coren ME, Meeks M, Buchdahl RM, et al. Primary ciliary dyskinesia (PCD) in children-age at diagnosis and symptom history. Acta Paediatr 2002;91:667-9.

4. Kennedy MP, Noone PG, Leigh MW, et al. High-resolution CT of patients with primary ciliary dyskinesia. Am J Roentgenol 2007;188:1232-8.

5. Meeks M, Bush A. Primary ciliary dyskinesia. Pediatr Pulmonol 2000;29:307-16.

6. Greenstone MA, Dewar A, Cole PJ. Ciliary dyskinesia with normal ultrastructure. Thorax 1983;38:875-6.

7. Greenstone $\mathbf{M}$, Rutman A, Pavia D, et al. Normal axonemal structure and function in Kartagener's syndrome: an explicable paradox. Thorax 1985;40:956-7.

8. Escudier E, Escalier D, Homasson JP, et al. Unexpectedly normal cilia and spermatozoa in an infertile man with Kartagener's syndrome. Eur J Respir Dis 1987;70:180-6.

9. Conraads VMA, Galdermans DI, Kockx MM, et al. Ultrastructurally normal and motile spermatozoa in a fertile man with Kartagener's syndrome. Chest 1992:102:1616-8.

10. Rutland J, de longh RU. Random ciliary orientation. A cause of respiratory tract disease. N Engl J Med 1990;323:1681-4.

11. Bush A, Ferkol T. Movement. The emerging genetics of primary ciliary dyskinesia. Am J Respir Crit Care Med 2006;174:109-11.

12. Pifferi M, Caramella D, Cangiotti AM, et al. Nasal nitric oxide in atypical primary ciliary dyskinesia. Chest 2007;131:870-3.

13. Lanz MJ, Prendes S, Peyrou N, et al. Nasal nitric oxide as a noninvasive marker in the antibiotic treatment of acute bacterial sinusitis. J Allergy Clin Immunol 2008;121:530-1.

14. Struben VM, Sewbalak WV, Wieringa MH, et al. Nasal nitric oxide in cystic fibrosis with and without humming. Eur J Clin Invest 2007;37:140-4.

15. Jorissen M, Willems T, Van der Schueren B, et al. Secondary ciliary dyskinesia is absent after ciliogenesis in culture. Acta Otorhinolaryngol Belg 2000;54:333-42.

16. Jorissen $\mathbf{M}$, Willems T, Van der Schueren B, et al. Ultrastructural expression of primary ciliary dyskinesia after ciliogenesis in culture. Acta Otorhinolaryngol Belg 2000:54:343-56.

17. Jorissen $\mathbf{M}$, Willems $\mathbf{T}$, Van der Schueren B. Ciliary function analysis for the diagnosis of primary ciliary dyskinesia: advantages of ciliogenesis in culture. Acta Otolaryngol 2000;120:291-5. 
18. Gamarra F, Bergner A, Stauss E, et al. Rotation frequency of human bronchial and nasal epithelial spheroids as an indicator of mucociliary function. Respiration 2006; 73:664-72.

19. Wald ER. Recurrent and nonresolving pneumoniae in children. Semin Respir Infect 1993;8:46-58.

20. Rutland J, Cole PJ. Non-invasive sampling of nasal cilia for measurement of beat frequency and study of ultrastructure. Lancet 1980;2:564-5

21. Braga PC. A variable-thickness, multipurpose culture chamber for high-magnification observation. J Microsc 1990;159:285-8.

22. Schidlow DV. Primary ciliary dyskinesia (the immotile cilia syndrome). Ann Allergy 1994;73:457-69.

23. Pifferi M, Cangiotti AM, Ragazzo V, et al. Primary ciliary dyskinesia: diagnosis in children with inconclusive ultrastructural evaluation. Pediatr Allergy Immunol 2001;12:274-82.

24. Rossman CM, Forrest JB, Lee RM, et al. The dyskinetic cilia syndrome. Ciliary motility in immotile cilia syndrome. Chest 1980;78:580-2.

25. Carlén B, Stenram U. Ultrastructural diagnosis in the immotile cilia syndrome. Ultrastruct Pathol 1987;11:653-8.
26. Baraldi E, de Jongste JC, European Respiratory Society/American Thoracic Society. Measurement of exhaled nitric oxide in children, 2001. Eur Respir J 2002;20:223-37.

27. Neugebauer $\mathbf{P}$, Endepols $\mathrm{H}$, Mickenhagen A. Ciliogenesis in submersion and suspension cultures of human nasal epithelial cells. Eur Arch Otorhinolaryngol 2003:260:325-30.

28. Jorissen M, Willems T. Success rates of respiratory epithelial cell culture techniques with ciliogenesis for diagnosing primary ciliary dyskinesia. Acta Otorhinolaryngol Belg 2000;54:357-65.

29. Kirkwood B, ed. Essentials of Medical Statistics. Oxford: Blackwell Scientific Publications, 1990.

30. Laoukili J, Perret E, Willems T, et al. IL-13 alters mucociliary differentiation and ciliary beating of human respiratory epithelial cells. J Clin Invest 2001;108:1817-24.

31. Jorissen M, Willems T, De Boeck K. Diagnostic evaluation of mucociliary transport: from symptoms to coordinated ciliary activity after ciliogenesis in culture. Am J Rhinol 2000;14:345-52.

32. Toskala E, Haataja J, Shirasaki H, et al. Culture of cells harvested with nasal brushing: a method for evaluating ciliary function. Rhinology 2005:43:121-4.

33. Bush A. How has research in the last five years changed my clinical practice? Arch Dis Child 2005;90:832-6. 